\title{
Human precision-cut liver tumor slices as a tumor patient-individual predictive test system for oncolytic measles vaccine viruses
}

\author{
MARTINA ZIMMERMANN ${ }^{1}$, SORIN ARMEANU ${ }^{1}$, IRINA SMIRNOW ${ }^{1}$, SUSAN KUPKA ${ }^{2}$, SILVIA WAGNER ${ }^{2}$, \\ MANFRED WEHRMANN ${ }^{3}$, MARIANNE G. ROTS ${ }^{4}$, GENY M.M. GROOTHUIS ${ }^{5}$, THOMAS S. WEISS ${ }^{6}$, \\ ALFRED KÖNIGSRAINER ${ }^{2}$, MICHAEL GREGOR $^{1}$, MICHAEL BITZER $^{1 *}$ and ULRICH M. LAUER ${ }^{1 *}$ \\ ${ }^{1}$ Department of Gastroenterology and Hepatology, Medical University Hospital, Otfried-Müller-Strasse 10; \\ ${ }^{2}$ Department of General, Visceral and Transplant Surgery, Medical University Hospital, Hoppe-Seyler-Strasse 3; \\ ${ }^{3}$ Department of Pathology, Medical University Hospital, Liebermeisterstrasse 8, 72076 Tübingen, Germany; \\ ${ }^{4}$ University of Groningen, University Centre for Pharmacy, Department of Therapeutic Gene Modulation, \\ P.O. Box 196, 9700 AD Groningen; ${ }^{5}$ University of Groningen, Department of Pharmacokinetics and Drug Delivery, \\ Antonius Deusinglaan 1, 9713 AV Groningen, The Netherlands; ${ }^{6}$ Center for Liver Cell Research, \\ University of Regensburg Hospital, F.-J.-S.-Allee 11, 93042 Regensburg, Germany
}

Received December 5, 2008; Accepted January 29, 2009

DOI: 10.3892/ijo_00000253

\begin{abstract}
Availability of an individualized preselection of oncolytic viruses to be used for virotherapy of tumor patients would be of great help. Using primary liver tumor resection specimens we evaluated the precision-cut liver slice (PCLS) technology as a novel in vitro test system for characterization of paramount tumor infection parameters of individual patients. PCLS slices from resection specimens of 20 liver tumor patients were cultivated in vitro for up to 5 days and infected with 5 different oncolytic measles vaccine virus $(\mathrm{MeV})$ strains. Effectiveness of tumor infection was monitored by viral nucleocapsid $(\mathrm{N})$ protein detection in immunofluorescence staining or Western blot analysis or by detection of GFP marker gene expression. MeV spreading in PCLS cultures was visualized by confocal microscopy. Oncolytic $\mathrm{MeV}$ vaccine particles were demonstrated to efficiently infect PCLS slices
\end{abstract}

Correspondence to: Dr Ulrich M. Lauer, University Hospital Tübingen, Otfried-Müller-Strasse 10, D-72076 Tübingen, Germany E-mail: ulrich.lauer@uni-tuebingen.de

*Share senior authorship

Abbreviations: HCC, hepatocellular carcinoma; CRC, colorectal carcinoma; PC, pancreatic carcinoma; CC, cholangiocarcinoma; PCLS, precision-cut liver slice; PHH, primary human hepatocytes; $\mathrm{MeV}$, measles vaccine virus; MOI, multiplicity of infection; CPE, cytopathic effect; dpi, days post infection

Key words: liver cancer, oncolytic viruses, measles vaccine viruses, precision-cut liver slices originating from different primary and secondary tumors of the liver with $\mathrm{MeV}$ strains Moraten/Edmonston Zagreb and AIK-C showing highest infection rates $(75 \%$ of all tested tumor specimens). Employing mixed liver tissue slices (exhibiting both tumorous and non-tumorous tissue areas on one and the same sample) a distinct tumor area favouring pattern of $\mathrm{MeV}$ infections was observed being in accordance with our finding that primary human hepatocytes are also permissive to $\mathrm{MeV}$ particles, albeit at a much lower rate and with a much less pronounced cytopathic effect. Furthermore, confocal microscopy demonstrated virus penetration throughout tumor tissues into deep cell layers. In conclusion, the PCLS technology is suitable to perform a tumor-patient individualized preselection of oncolytic agents prior to clinical virotherapeutic applications.

\section{Introduction}

Since early case studies emphasized regression of cancers during naturally acquired virus infections, viruses have attracted considerable interest as possible agents of tumor destruction (1) as well as personalized cancer vaccines (2). Such oncolytic viruses have a great potential to complement many of today's standard cancer therapies (3). As safety of replication-competent viral vectors is of utmost importance, especially vaccine virus strains exhibiting a long-standing and well proven safety record (4) purposefully have been chosen for future virotherapeutic approaches (5).

Measles vaccine viruses $(\mathrm{MeV})$ are characterized by human apathogenicity and by exhibiting a potent natural oncolytic activity (6-11) and safety profile (12). In the past, a large variety of different $\mathrm{MeV}$ vaccine strains has been generated (reviewed in ref. 13), including strains Mérieux/ Schwarz, Moraten/Edmonston Zagreb, Leningrad-16 (L-16), and AIK-C. Furthermore, recombinant $\mathrm{MeV}$ vectors encoding 
marker genes like the green fluorescent protein (MeV-GFP) have been generated (14). However, so far no platform for a comprehensive comparison of the oncolytic effectiveness of closely related, but distinctive measles vaccine viruses has been provided. Up to now, only human tumor cell lines are in use as the standard system to test and compare the efficacy of various oncolytic viruses. Consequently, there is not much knowledge on the possible side effects in human nontumorous tissues neighboring primarily addressed tumor nodules. Such safety investigations require i) the direct use of primary human tissues (encompassing both tumorous as well as adjacent non-tumorous tissue sections), ii) means for their prolonged in vitro culture as well as iii) capabilities for monitoring paramount tumor infection parameters.

The precision-cut liver slice (PCLS) technique is capable of generating and cultivating slices of 200-300 $\mu \mathrm{m}$ thickness [exhibiting 10-15 cell layers of human tumorous and nontumorous tissues; (15-17)] accessible for viral infections (18-20). By this technique, individual patient features such as extracellular matrix composition with Kupffer cell and stellate cell functions, which are important to the normal function of the hepatocyte unit, are preserved $(21,22)$. In addition, PCLS maintain the heterogeneity and complex phenotype of individual tumors, including the vasculature (18).

In this study, our aim was to evaluate usage of the PCLS system as a predictive test system for the oncolytic effectiveness of different measles vaccine viruses in primary or secondary tumors of the human liver. Besides a detailed molecular analysis, formation of $\mathrm{MeV}$-induced syncytia (multinucleated non-viable giant cells resulting from fusion of infected cells with neighboring cells) was taken as a prominent feature of target cell infection.

\section{Materials and methods}

Cells. African green monkey kidney cells (Vero), human hepatoma cells ( $\mathrm{HuH} 7)$, human colorectal carcinoma cells (HT29) and human pancreatic carcinoma cells (MiaPaCa) were grown at $37^{\circ} \mathrm{C}$ in Dulbecco's modified Eagle medium (DMEM; Lonza Bioscience, Verviers, Belgium) supplemented with $10 \%$ fetal calf serum (FCS; Perbio Science, Bonn, Germany), in a humidified atmosphere of $5 \% \mathrm{CO}_{2}$. Primary human hepatocytes $(\mathrm{PHH})$ were isolated and kindly provided by T.S. Weiss and cultured as described previously (23). Experimental procedures were performed according to the guidelines of the Ethics Committee, University Hospital of Regensburg, with informed consent. The tissues were screened by the Human Tissue and Cell Research foundation (HTCR) (24).

Human tissues. Human liver resection material from 20 donors containing primary and secondary liver tumors surrounded by non-tumorous liver tissue was obtained according to the guidelines of the local Ethics Committee with informed consent by the Department of General, Visceral and Transplant Surgery, University of Tübingen. Explanted tissues were stored immediately in ice-cold William's E medium (Lonza Bioscience, Verviers, Belgium) and transported directly to the laboratory where the slicing procedure started within $1 \mathrm{~h}$.
Slicing. Precision-cut tissue slices were prepared out of tissue cores of $8 \mathrm{~mm}$ diameter by employing a special coring device (Alabama Research and Development, Munford, USA). The tissue cores were cut under buffered conditions with ice-cold oxygen-saturated Krebs-Henseleit buffer (KHB) containing $25 \mathrm{mM}$ glucose (Merck, Darmstadt, Germany), 25 mM NaHCO 3 (Roth, Karlsruhe, Germany) and $10 \mathrm{mM}$ HEPES (Roth) by using the Krumdieck Tissue Slicer (Alabama Research and Development) as described previously $(25,26)$. Thereby, slices with $8 \mathrm{~mm}$ in diameter and a thickness of 200-300 $\mu \mathrm{m}$ (containing $1 \times 10^{6}$ cells in average) were produced and collected in ice-cold KHB.

Cultivation of tissue slices. After slicing, samples were washed with oxygenated William's E medium supplemented with $25 \mathrm{mM}$ glucose and $50 \mu \mathrm{g} / \mathrm{ml}$ gentamycin (WEGG) and transferred to 12 -well plates. Cultivation was performed in $2 \mathrm{ml}$ WEGG medium for each slice under a highly oxygenated atmosphere $\left(80 \%\right.$ oxygen, $5 \% \mathrm{CO}_{2}$ ) at $37^{\circ} \mathrm{C}$ with gentle shaking.

Virus strains. In this study, we used the following measles vaccine $(\mathrm{MeV})$ strains: Mérieux/Schwarz strain (Sanofi Pasteur MSD GmbH, Leimen, Germany), Moraten/Edmonston Zagreb strain (Berna Biotech AG, Bern, Switzerland), Leningrad-16 strain (a kind gift from the Robert Koch-Institut, Berlin, Germany), AIK-C strain (Hokken, Kitasato, Japan). Recombinant measles virus encoding the GFP marker gene [MeV-GFP; (14)] was kindly provided by S.J. Russell, Mayo Clinic, Rochester, MN, USA. For virus production $1 \times 10^{7}$ Vero cells were plated in 14-cm diameter dishes, infected with $\mathrm{MeV}$ particles at a multiplicity of infection (MOI) of 0.01 in serum-free medium (X-Vivo, BioWhittaker, Walkersville, MD) for $1 \mathrm{~h}$ and grown at $33^{\circ} \mathrm{C}$ in DMEM containing $2 \% \mathrm{FCS}$ in a humidified atmosphere of $5 \% \mathrm{CO}_{2}$ until the infection was spread to $>80 \%$ of cells. Next, supernatants were discarded; the cells were washed with PBS, then covered with $1.5 \mathrm{ml}$ $\mathrm{X}$-Vivo and frozen at $-80^{\circ} \mathrm{C}$ overnight. After thawing, the cells were pressed through a needle 15 times to release the virus particles into the supernatant. For removal of the cell debris, the suspension was centrifuged at $4,000 \mathrm{x}$ g for $5 \mathrm{~min}$. The resulting virus solution was frozen in $100 \mu \mathrm{l}$ aliquots at $-80^{\circ} \mathrm{C}$. Finally, virus titers were determined using the $\mathrm{TCID}_{50}$ method (27). The identity of the different vaccine viruses was proven by sequencing a part of the viral M-gene amplified by RT-PCR using specific forward (5' tgcctcccaagttccacaat $3^{\prime}$ ) and reverse ( $5^{\prime}$ tggectctttgaggactttttc $3^{\prime}$ ) primers. For viability control of human tissue slices, a non-replicating GFP marker gene expressing recombinant adenovirus (AdV) was used (28).

Infection of cells and slices. Cells were infected with $\mathrm{MeV}$ in $\mathrm{X}$-Vivo serum-free medium for $1 \mathrm{~h}$ at $37^{\circ} \mathrm{C}$ at defined MOIs. Following incubation, virus solutions were removed and fresh culture medium was added. Slices were infected 1 day postslicing with $10^{6} \mathrm{TCID}_{50}$ of $\mathrm{MeV}$ or $10^{8} \mathrm{TCID}_{50}$ of $\mathrm{AdV}$ in $500 \mu \mathrm{l}$ WEGG culture medium for $1 \mathrm{~h}$ at $37^{\circ} \mathrm{C}$ under an oxygenated atmosphere ( $80 \%$ oxygen, $5 \% \mathrm{CO}_{2}$ ). Following incubation, virus solutions were removed and $2 \mathrm{ml}$ of fresh culture medium was added. 
Immunocytochemistry and microscopy. Slices or cells were fixed with $4 \%$ formaldehyde solution (Fischar $\mathrm{GmbH}$, Saarbrücken, Germany) overnight at $4^{\circ} \mathrm{C}$, washed with PBS and blocked with Tris-buffered saline with $0.2 \%$ Tween-20 and $1 \%$ FCS. Virus detection was performed using a primary mouse anti-measles nucleocapsid protein antibody (1:1000, NP cl. 120, ECACC, Porton Village, UK) followed by a secondary antibody goat anti-mouse IgG $(\mathrm{H}+\mathrm{L})$ coupled to Alexa Fluor 546 (1:1000, Molecular Probes, Invitrogen $\mathrm{GmbH}$, Karlsruhe, Germany). Nuclear staining was performed with $1 \mu \mathrm{g} / \mathrm{ml}$ DAPI (Sigma-Aldrich, Munich, Germany) for fluorescent microscopy and with Sytox green (Molecular Probes) diluted 1:50,000 for confocal microscopy. Pictures were taken with an Olympus IX50 fluorescence microscope using analysis 3.1 software (Soft Imaging System GmbH, Münster, Germany). Confocal z-stack pictures were generated with a Zeiss confocal microscope (Axiovert 200M) and the LSM 510 3.2 SP software (Carl Zeiss MicroImaging GmbH, Göttingen, Germany). For this purpose, slices were put on a microscope slide in a plane orientation. To scan a complete tissue slice from the upper to the lower surface and beyond, 80 stack-scanned pictures with a z-stack size of $3.05 \mu \mathrm{m}$ were required. A virtual 3D view of one slice was calculated with IMARIS 4.0 software (Bitplane scientific solutions, Zürich, Switzerland).

Western blot assays. To detect viral protein, $500 \mu 1$ DMEM was added to each frozen tissue slice and the tissue was treated with ultrasound using a high intensity cup horn sonifier (Branson, Danbury, CT, USA) for $30 \mathrm{sec}$ at maximum power. Proteins from $50 \mu \mathrm{l}$ of each sample were electrophoretically separated on a $10 \%$ SDS polyacrylamide gel and subsequently transferred to a polyvinylidene difluoride membrane (Highbond P; Amersham Biosciences, Freiburg, Germany) as described previously (29). For detection of specific proteins a rabbit anti-measles nucleoprotein $(\mathrm{N})$ antibody $(1: 5,000$; ab23974, Abcam, Cambridge, UK) and a mouse anti-vinculin antibody (1:5,000; V-9131, Sigma-Aldrich) were employed. Secondary antibodies against rabbit $(1: 30,000 ; 170-6515$ Bio-Rad, Munich, Germany) and mouse (1:30,000; 170-6516, Bio-Rad) coupled to HRP were used.

Histological analysis. Liver and tumor tissues and cultivated tissue slices were fixed in $4 \%$ buffered formalin for at least $24 \mathrm{~h}$ and embedded in paraffin. Serial sections were performed and stained with H\&E. The histological analyses including the tissue status (viability, inflammation processes) as well as morphological features (differentiation into tumorous and non-tumorous liver areas, necrosis, fibrosis, cirrhosis) were performed by an expert pathologist.

\section{Results}

Analyses of human primary and secondary liver tumor resection materials. Our study encompasses resection materials from 20 human donors exhibiting primary $(8 \mathrm{HCC}$, $3 \mathrm{CC}$ ) or secondary (9 CRC) tumors of the liver.

First, careful macroscopical examinations were carried out to define tumorous, non-tumorous, as well as mixed tumorous/ non-tumorous tissue areas being suitable for subsequent
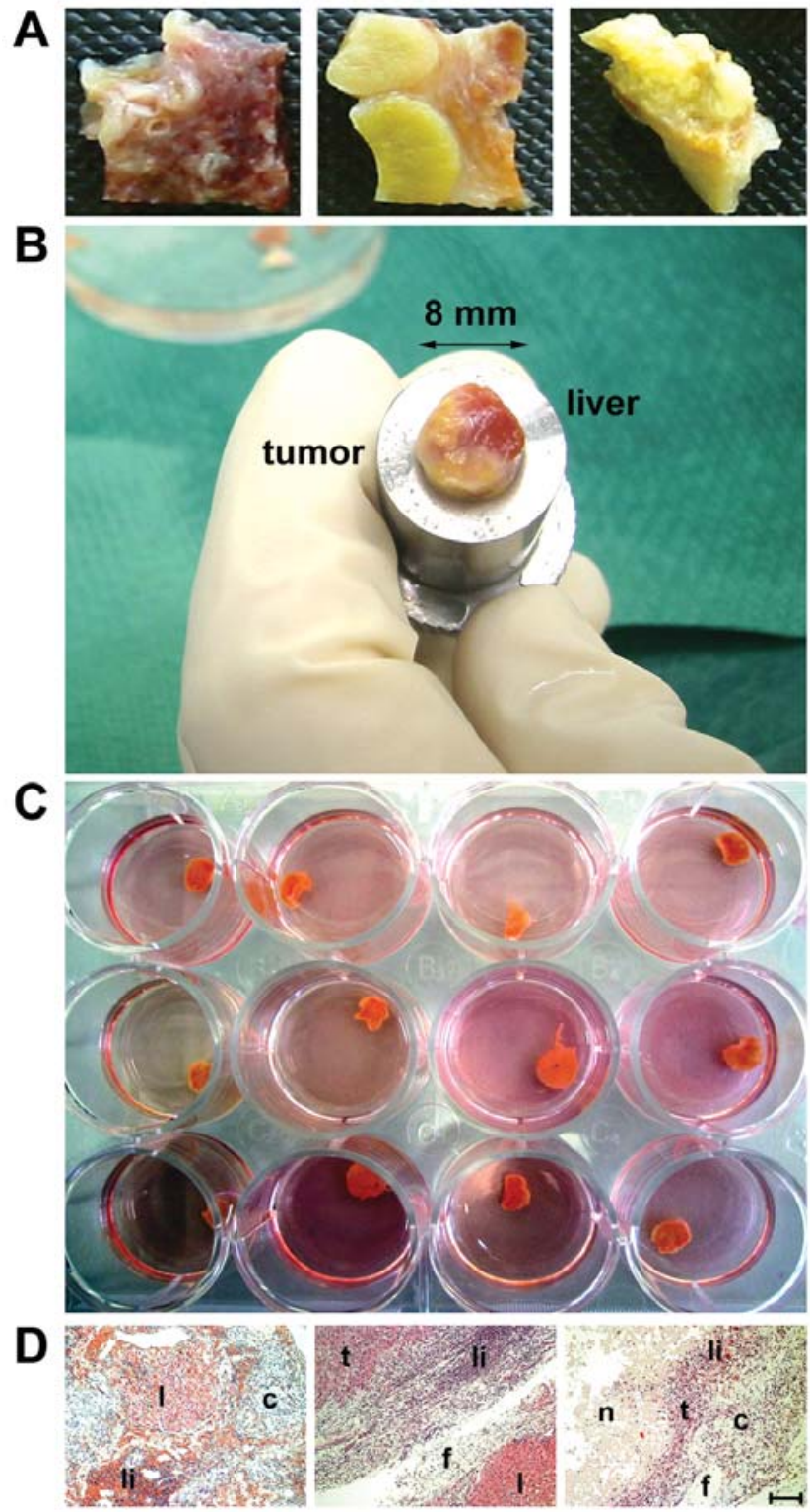

Figure 1. Precision-cut slicing of resection material from a patient with hepatocellular carcinoma (HCC). (A) Macroscopical view. Tissue samples from an HCC patient are composed mainly of non-tumorous liver (left), of two yellowish tumor nodules clearly separated from reddish non-tumorous liver tissue (middle) and of predominantly necrotic HCC tissue (right). (B) For slicing, cylinders of $8 \mathrm{~mm}$ diameter were punched out of a mixed tumorous/non-tumorous tissue sample [middle panel in (A)]. (C) After fixation in a microtome cylinder, precision-cut liver slices were prepared, preincubated for $1 \mathrm{~h}$ in fresh WEGG medium and than placed into 12-well plates (one slice per well containing $2 \mathrm{ml}$ of WEGG medium) and cultivated for up to 5 days in highly oxygenated WEGG medium (upper panel). (D) Freshly isolated slices were fixed in formalin, embedded in paraffin, cut in 5 $\mu \mathrm{m}$ thin sections followed by HE staining. Histological analysis revealed major variations concerning both the composition as well as the integrity of sliced tissues; visible are parenchymatous liver (l) as well as tumor (t) cells, lymphocytic infiltrates (li), fibrotic (f), necrotic (n) and cirrhotic (c) areas; bar represents $100 \mu \mathrm{m}$.

punching. As an example, resection material from a patient with HCC (Fig. 1A) contained tumor areas which mainly exhibited a yellowish colour (right panel), whereas surrounding non-tumorous fibrotic and cirrhotic liver tissue displayed a reddish colour (left panel). This divergence in macroscopical appearance was pronounced for resection samples being 
composed of mixed tumorous/non-tumorous areas (Fig. 1B; punch, origination from the resection material displayed in Fig. 1A, middle panel). In general, vitality of resection material is known to differ due to features such as i) pre-treatment with classical chemotherapeutics, biologicals or radiation, ii) central necrosis in large tumors, or iii) prolonged resection surgery procedures; thereby, the typical patient heterogeneity of study cohorts employing investigational new drugs was also reflected in our tissue slice study.

Following this initial macroscopical analysis, precision-cut liver slices (PCLS) were generated, assembled and cultured for up to 5 days in 12-well plates (Fig. 1C). As a result, selected slices encompassing tumorous or non-tumorous areas respectively, or mixed (tumorous and non-tumorous) areas were obtained; macroscopical assignments were confirmed histologically (Fig. 1D) by an expert pathologist. For example, the reddish non-tumorous tissue sample (Fig. 1A, left panel) showed a cirrhotic liver tissue with some lymphocytic infiltrates (Fig. 1D, left panel), the sample being composed of mixed tumorous/non-tumorous (yellowish/ reddish) areas (Fig. 1A, middle panel) revealed both liver and tumor cells containing fibrotic areas (Fig. 1D, middle panel), and the lightly yellowish fluffy tissue sample (Fig. 1A, right panel) displayed a mostly necrotic tumor area with some fibrotic and cirrhotic segments (Fig. 1D, right panel). Therefore, macroscopic classification correlated well with subsequent histological findings.

Measles vaccine virus infection of human liver tumor cells of hepatobiliary and gastrointestinal origin. We then set out to compare the infectability of tumor cells of hepatobiliary and gastrointestinal origin by oncolytic measles vaccine viruses $(\mathrm{MeV})$ on i) human tumor cell lines of clonal origin cultured as monolayers versus ii) human donor derived multilayer slices representing all heterotypic features of naturally emerging primary and secondary liver tumors.

First, human HuH7 hepatoma cells (HCC), human HT29 colorectal carcinoma cells (CRC), as well as human MiaPaCa pancreatic carcinoma cells (PC) were infected with recombinant GFP marker gene expressing measles viruses (MeV-GFP) at a multiplicity of infection (MOI) of 1 (Fig. 2A). As a result, $\mathrm{HuH7}$ hepatoma cells not only showed the fastest progression of infection [Fig. 2A, third column (HCC), upper panel: cells exhibiting a maximum of green fluorescencepositive syncytia at 2 days post infection (dpi)], but also the strongest effect of tumor cell lysis [Fig. 2A, third column (HCC), lower panels: tumor cell lysis was almost complete at 3 dpi (phase contrast image) with only a few GFP-positive syncytia being remnant at this time point (fluorescence picture)].

This MeV-GFP-induced cytopathic effect (CPE) was comparable to the CPE observed in $\mathrm{MeV}$ producer cells (Vero) which are known to be highly permissive for infections with $\mathrm{MeV}$ [Fig. 2A, first column (Vero): extensive syncytia formation and almost complete cell lysis at $3 \mathrm{dpi}$.

Human HT29 colorectal carcinoma cells (CRC) and human MiaPaCa pancreatic carcinoma cells (PC) also were found to be strongly infected and lysed by MeV-GFP [Fig. 2A, last two columns (CRC, PC)]. As a control for viral accessibility of non-transformed parenchymatous cells of the liver, primary human hepatocytes $(\mathrm{PHH})$ from several different donors were infected with $\mathrm{MeV}$-GFP. In a representative donor, only some rare events of infection by MeV-GFP became visible at 2 dpi [Fig. 2A, second column (PHH)]; at 3 dpi, very few syncytia had developed in the PHH cell layer, but, in comparison to all tested tumor cell lines, no signs of gross cellular destruction were visible in phase contrast imaging [Fig. 2A, second column (PHH)] or in an LDH leakage and SRB viability assay (data not shown). This indicates that $\mathrm{PHH}$ actually are permissive to $\mathrm{MeV}$ particles, albeit at a much lower rate and with a much less pronounced CPE than tumor cell lines.

Further on, we assessed whether primary human liverderived tumor tissues are susceptible to measles vaccine virus. For this purpose, we infected liver tumor tissue slices of different origin at a MOI of 1 with MeV-Moraten/Edmonston Zagreb (Fig. 2B: depiction of representative samples of HCC, $\mathrm{CRC}$, and CC). The slices were fixed with formaldehyde $4 \mathrm{dpi}$ and viral $\mathrm{N}$ protein was detected by immunofluorescence staining (Fig. 2B, bright red colour, marked by white arrows). Virus-positive regions in tissue slices were found to be extended over a distance of $500 \mu \mathrm{m}$ on average. This observation was similiar to cellular fusions that occur during an early stage of infection in monolayer cell cultures (Fig. 2A). Thus, oncolytic measles vaccine virus was demonstrated to infect human tissues of different primary and secondary tumors of the liver efficiently.

Patterns of virus distribution in liver tumor tissue slices. Next, we assessed if there is a strong infection preference of oncolytic measles vaccine virus for tumor cells not only in monolayer cell cultures (Fig. 2A), but also in the context of liver tumor tissue slices.

For this purpose, mixed liver tissue slices were generated exhibiting both tumorous and non-tumorous tissue areas on one and the same sample (example depicted in Fig. 1B). In general, both - human liver tissue as well as primary tumors of the liver - revealed a strong autofluorescence. In contrast, secondary tumors of the liver had much less background in fluorescent light (Fig. 3).

Therefore, mixed (tumorous/non-tumorous) liver tissue slices exhibiting secondary liver tumors were infected with recombinant MeV-GFP particles at a MOI of 1 . Fluorescent microscopy pictures were taken as before. To obtain a 'panoramic' view covering a complete tissue slice, 20 fluorescent microscopy pictures from one slice were combined to a single photograph. Fig. 3 shows a mixed tissue slice composed of a colorectal liver metastasis (displayed in dark green due to low grade autofluorescence) and a large margin of surrounding non-tumorous liver tissue (with intense light green autofluorescence; tumor/non-tumor boundary marked by yellow arrows). As a result, a distinct tumor area favouring GFP marker gene expression, reflected by formation of large GFP-positive syncytia, was observed at 3 dpi.

Successful future clinical applications require an almost complete tumor cell lysis. Thus, virus spread into deeper cell layers inside the tumor will be of utmost importance. Since this important property of virotherapeutics can only be analyzed in multilayer tissue specimens, human tissue slices 

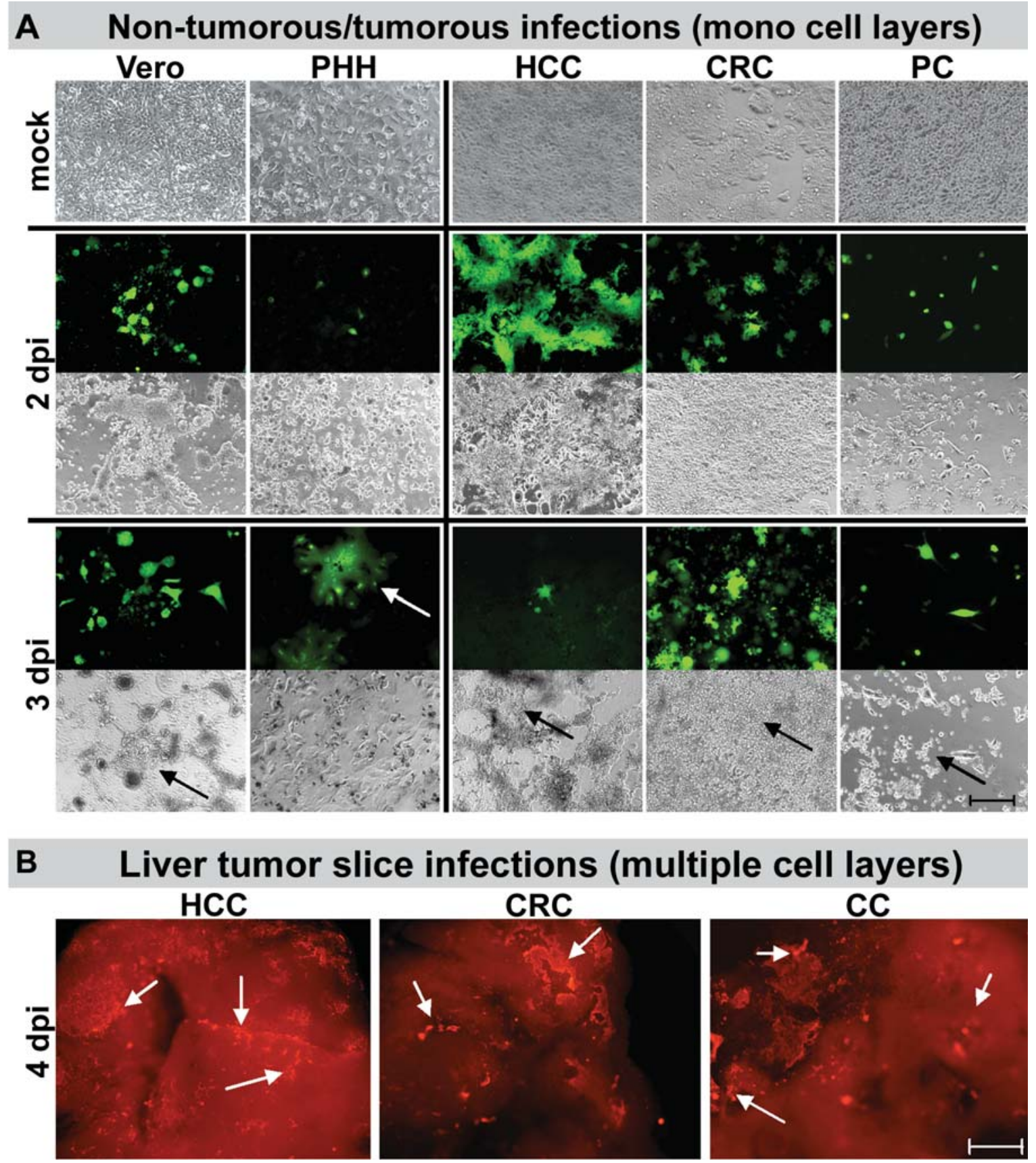

Figure 2. Measles vaccine viruses (MeV) not only infect cell lines of non-tumorous and tumorous origin, but also tissue slices originating from different human tumors. (A) Monkey kidney cells (Vero), primary human hepatocytes (PHH), human HuH7 hepatoma cells (HCC), human HT29 colorectal carcinoma cells (CRC) and human MiaPaCa pancreatic carcinoma cells (PC) were infected with recombinant GFP expressing measles virus (MeV-GFP). Pictures were taken 2 and 3 days post infection (dpi) (upper panels: cells infected with GFP expressing MeV are visualized by green fluorescence; lower panels: phase contrast microscopy pictures). Mock pictures were taken at $2 \mathrm{dpi}$. MeV was found to infect Vero, HuH7, and MiaPaCa cultures much faster than primary human hepatocytes or HT29 tumor cells. On 3 dpi, PHH only show single syncytia formations (white arrow), whereas gross destruction of infected cells was observed for Vero, HuH7, HT29, and MiaPaCa cells (black arrows). Bar represents $200 \mu \mathrm{m}$. (B) Liver slices from 3 human donors with HCC, CRC, and cholangiocarcinoma (CC) were infected with non-recombinant $\mathrm{MeV}$ (Moraten/Edmonston Zagreb strain). Formalin fixation and immunofluorescence staining of MeV $\mathrm{N}$ (nucleocapsid) protein were performed 4 dpi. Viral infections are reflected by a bright red staining; areas of major infection spots are indicated by white arrows. Bar represents $500 \mu \mathrm{m}$

are considered as an ideal system for this purpose. To visualize virus spread throughout tumor tissue, we used a laser scan microscope. Confocal microscopy technology enables us to look into defined tissue layers, which definitely are not accessible using a standard fluorescence microscope.

Again, tissue slices from a human donor with colorectal liver metastasis were infected with a MeV vaccine virus (strain
Moraten/Edmonston Zagreb, MOI of 1) and fixed in formalin four days later. Virus detection was performed using a primary mouse anti-MeV $\mathrm{N}$ antibody and a secondary anti-mouse antibody coupled to Alexa Fluor 546. All cells were visualized by an unspecific nucleic acid staining with Sytox green. By combining 80 single pictures with a height of each $3.05 \mu \mathrm{m}$ to a virtual 3D model, the surface and the structure of individual tissue slice clippings became visible (Fig. 4A). 


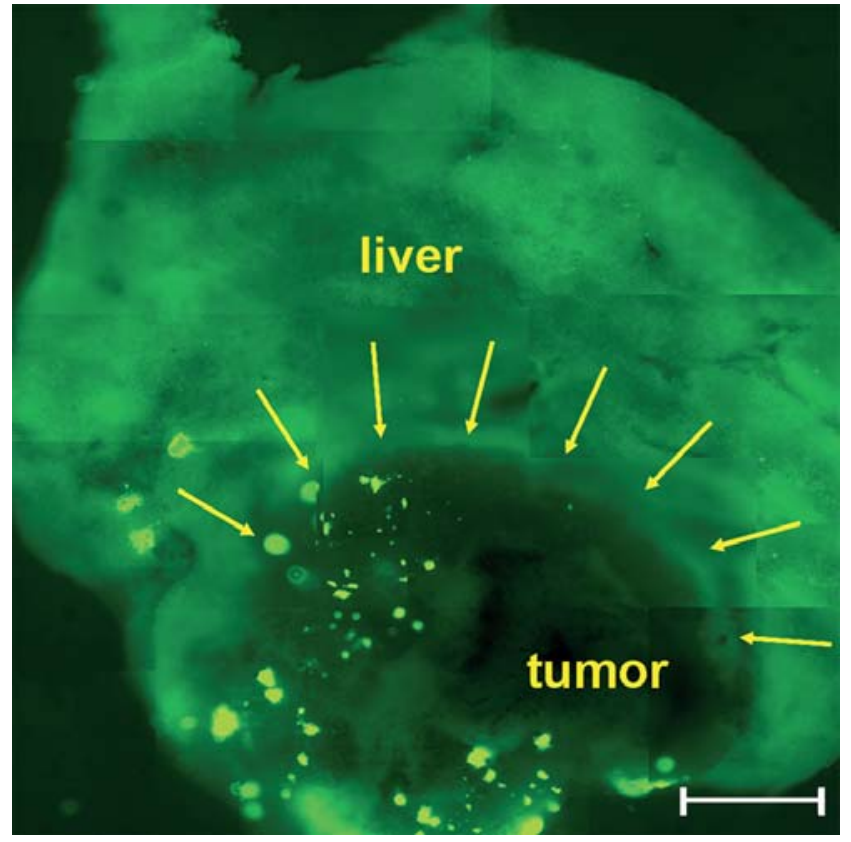

Figure 3. A mixed, non-tumorous/tumorous liver slice infected with MeV-GFP displays a tumor area favouring marker gene expression. A precision-cut liver slice obtained from a patient exhibiting a colorectal liver metastasis was infected with MeV-GFP (MOI of 1) followed by fluorescence microscopy on day 3 post infection (20 single pictures were taken and combined to obtain one picture). The tumor area can be discriminated from surrounding normal, non-tumorous liver tissue (liver) by its dark ground colour (tumor margins are depicted by arrows); light green color represents spots of viral infection visualized by strong expression of the GFP marker gene. Bar represents $1 \mathrm{~mm}$.

Furthermore, $\mathrm{MeV}$ infection (displayed in brown colour) had spread throughout the tissue slice and the virus interpenetrated the whole tissue (Fig. 4B). To show virus spread in detail, virtual cross sections along the $y-z$ plane and the $x-y$ plane were placed into the simulated tissue model (Fig. 4C). Indeed, expression of measles virus $\mathrm{N}$ protein (displayed in a red/yellow colour) could be detected at plentiful spots deep within the tissue specimen (Fig. 4D); in contrast, uninfected cells remained green. Thereby, the ability of $\mathrm{MeV}$ to penetrate into deeper cell layers of tumor specimens of distinct patients could have been demonstrated successfully.

Infection depends on tissue quality and virus strain. Variations in quality of tissues obtained from surgical resections require a fast, easy and inexpensive quality check for each and every donor sample, so that samples with insufficient viability and consecutively insufficient infectability can be excluded as early as possible from further processing.

Since only viable cells provide the possibility for infection and subsequent expression of virus-encoded marker genes, we used a highly infectious replication-deficient recombinant adenovirus encoding the GFP marker gene (AdV-GFP; MOI of 100) to obtain a quick and conclusive read-out on the viability features of freshly sliced tissues; only tissue slices being positive for adenoviral-based expression of GFP at 1 day post infection (Fig. 5B; right pictures) were taken for further evaluation; as replication-deficient AdV-GFP particles are not able to spread to neighbouring cells, only primary adenoviral infections could take place providing an exact idea about
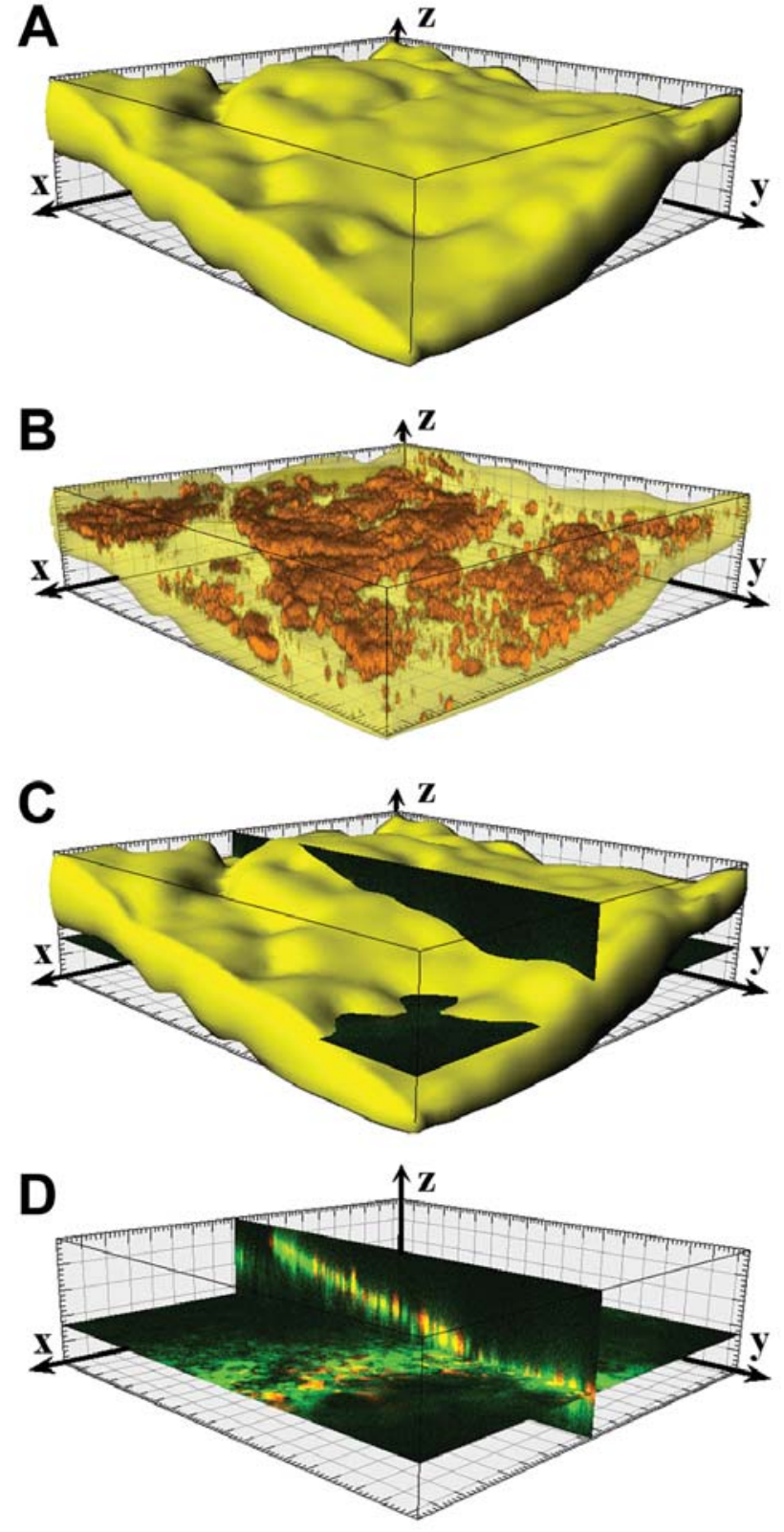

Figure 4. MeV penetrates into deep layers of tumorous liver tissue slices. A tissue slice from a human donor with colorectal liver metastasis was infected with $\mathrm{MeV}$ (Moraten/Edmonston Zagreb strain, MOI of 1). Formalin fixation and immunofluorescence staining of $\mathrm{MeV} \mathrm{N}$ (nucleocapsid) protein were performed 4 days post infection, followed by confocal immunofluorescence microscopy. (A) 3D model: the structure of a representative tumorous liver tissue slice (cut-out: x/y orientation $921 \mathrm{x} 921 \mu \mathrm{m}$, total height $240 \mu \mathrm{m}$, mean tissue slice thickness here: $150 \mu \mathrm{m}$ ) was calculated out of 80 single confocal pictures using the IMARIS 4.0 software. One square represents $50 \mu \mathrm{m}$. (B) Internal infections: $\mathrm{MeV}$ infections (brown) are visualized in total throughout the tissue. (C) Section setting: a virtual cross section along the $y$ $\mathrm{z}$ plane and a plane section along the $\mathrm{x}-\mathrm{y}$ plane was placed into the simulated tissue. (D) Infection details: MeV infected cells (indicated by red/yellow colour) are observed even in the depth of the tumorous liver tissue slice; uninfected cells are visible by their unspecific staining with Sytox green (green).

the actual condition of each tissue sample used for further processing.

Consequently, only viable tissue slices from several HCC donors [as demonstrated by Ad-GFP-positive signals (Fig. 5B, right column)] were then employed to compare the oncolytic 


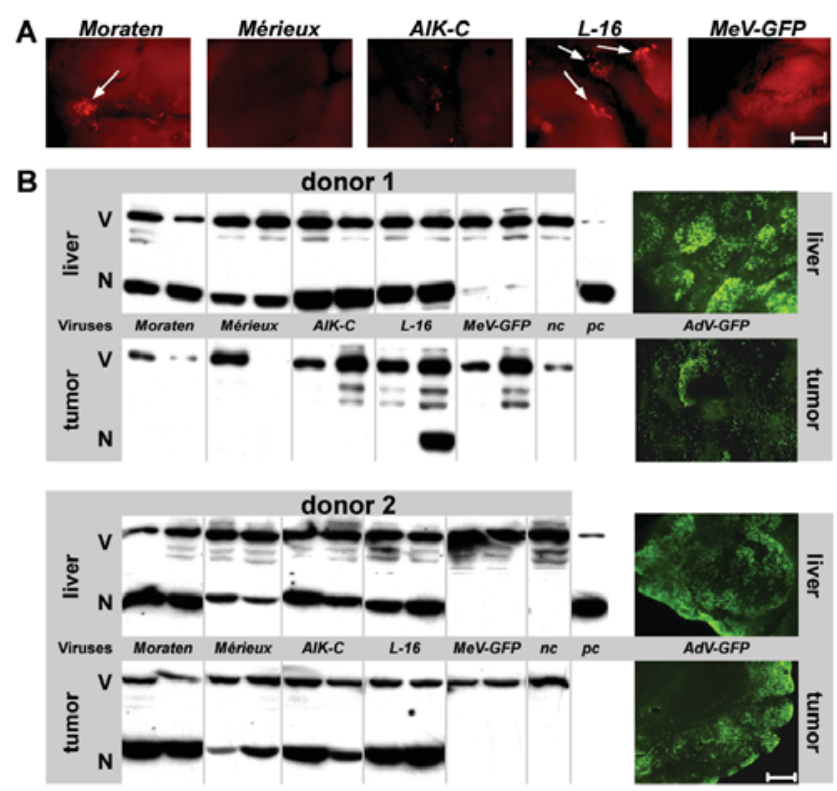

Figure 5. Efficiency of tissue slice infection employing different $\mathrm{MeV}$ vaccine strains. (A) Tissue slices from a patient with hepatocellular carcinoma (HCC) were infected with different $\mathrm{MeV}$ vaccine strains (MOI of 1). Immunofluorescence staining of $\mathrm{MeV} \mathrm{N}$ (nucleocapsid) protein was performed 4 days post infection. Viral infections are visualized by bright red staining; dark red staining represents background fluorescence (white arrows: major infection spots). Slices infected with $\mathrm{MeV}$ vaccine strains Moraten Edmonston Zagreb and Leningrad-16 (L-16) displayed large spots of MeV infection, whereas slices infected with strain AIK-C displayed smaller spots. On slices infected with Mérieux/Schwarz and MeV-GFP no infection could be observed at all. Bar represents $200 \mu \mathrm{m}$. (B) Left panel: Infections of nontumorous liver slices (liver) as well as tumorous tissue slices (tumor) from two $\mathrm{HCC}$ patients (donors 1+2) were performed with different $\mathrm{MeV}$ vaccine strains (Moraten/Edmonston Zagreb, Mérieux/Schwarz, AIK-C, Leningrad (L-16), and MeV-GFP) in duplicates (two lanes per strain). Infection efficiency was estimated by Western blot analysis of $\mathrm{MeV} \mathrm{N}$ protein $(\mathrm{N}$ ) detection of Vinculin (V) served as a loading control. Middle panel: Lysates from uninfected Vero cells (nc-negative control) or Vero cells infected with $\mathrm{MeV}$ vaccine strain Moraten/Edmonston Zagreb (pc-positive control) were used as controls for expression of $\mathrm{MeV} \mathrm{N}$ protein, respectively. Right panel: For an independent control of tissue slice cellular integrity over the whole time span of cultivation, infections with a replication-deficient GFP marker gene encoding adenovirus (AdV-GFP) were performed on selected slices of donors $1+2$. Fluorescence microscopy pictures taken on day one post infection showed that expression of the GFP marker gene displayed a heterogeneous distribution pattern over large areas of the infected tissue slices as revealed by the bright green fluorescence, thereby proving both, infectability and integrity of the infected slices. Bar represents $500 \mu \mathrm{m}$

potency of four different measles vaccine viruses (strains Moraten/Edmonston Zagreb, Mérieux/Schwarz, AIK-C, and L-16) and the recombinant MeV-GFP vaccine virus (Fig. 5).

For this purpose, tumor slices from one donor were fixed in formalin 4 days post infection and viral $\mathrm{N}$ protein was detected by immunofluorescence staining (Fig. 5A). As a result, tumor slices infected with $\mathrm{MeV}$ vaccine viruses Moraten/Edmonston Zagreb and L-16 showed large spots of $\mathrm{MeV}$ infection (Fig. 5A, bright red spots in panels 1 and 4, marked by white arrows). In contrast, in AIK-C infected tissues only small centres of infection could be observed (Fig. 5A, middle panel). Tumor slices infected with $\mathrm{MeV}$ Mérieux/Schwarz and MeV-GFP did not show any infections at all (Fig. 5A, panels 2 and 5). This observation points out that some MeV strains are suitable to infect tumor cells of a given patient, but others not.

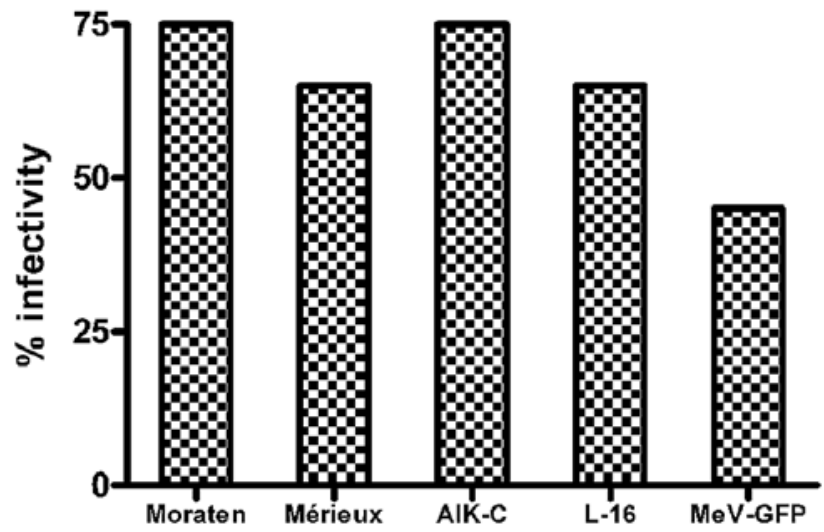

Figure 6. $\mathrm{MeV}$ infection rates in tumorous tissue slices. For a comparison of infection rates with $\mathrm{MeV}$ vaccine strains Moraten/Edmonston Zagreb, Mérieux/Schwarz, AIK-C, Leningrad (L-16) and MeV-GFP (MOI of 1) Western blot results of samples obtained from 20 donors exhibiting primary or secondary tumors of the liver ( $8 \mathrm{HCC}, 3 \mathrm{CC}, 9 \mathrm{CRC}$ ) were analyzed. For each donor we infected two slices for each virus strain. Even a singlepositive signal was interpreted as a positive event for a virus/donor combination. Therefore, it was unimportant whether both or only one slice gave a positive signal in Western blot detection. Correspondingly, a negative event occurred, when both slices from one donor infected with the same virus were negative. The data were analyzed by a proportional calculation between positive and negative events. Highest overall infectivity was reached by non-recombinant $\mathrm{MeV}$-Moraten/Edmonston Zagreb and $\mathrm{MeV}$ AIK-C vaccine strains (75\%); the lowest infection rate was observed with the recombinant marker gene encoding vector MeV-GFP (45\%).

Reflecting commonly observed differences in the viability status of the patient liver samples, donor 1 showed an intense AdV-GFP infection of the non-tumorous liver tissue (Fig. 5B, upper right panel, light green spots), whereas the tumorous tissue was much less infected by AdV-GFP (Fig. 5B, second panel from top, exhibiting much less light green spots). This unequal and discrepant infectability of the non-tumorous versus tumorous liver tissue by AdV could also be observed for infections with $\mathrm{MeV}$ as being reflected by the results of a Western blot analysis for MeV N protein expression (Fig. 5B, upper left panel): in non-tumorous tissue slices, $\mathrm{MeV} \mathrm{N}$ protein detection $(\mathrm{N})$ was successful for all four $\mathrm{MeV}$ vaccine strains (Moraten/Edmonston Zagreb, Mérieux/Schwarz, AIK-C, L-16; Fig. 5B, upper left, liver panels), but a rare event in the tumorous tissue slices, except for the detection of a positive signal in one of the tumorous tissue slices infected with L-16 (Fig. 5B, upper left, tumor panels). Thus, low AdV-GFP infectability came along with low MeV infectability and low or no expression of $\mathrm{MeV} \mathrm{N}$ protein.

In contrast, tissue slices from HCC donor 2 (Fig. 5B, lower left panels) demonstrated a positive pattern of $\mathrm{MeV}$ infections throughout both non-tumorous (liver) and tumorous (tumor) slices; this result came along with the positive pattern of AdV viability test infections in both non-tumorous and tumorous slices performed in parallel (Fig. 5B, lower right panels).

Next, by calculating the rate of $\mathrm{MeV}$ infection for all 20 donors with $\mathrm{HCC}, \mathrm{CC}$ and $\mathrm{CRC}$ tumors, we assessed which $\mathrm{MeV}$ vaccine strain showed the highest rate of overall infectivity of tumorous target tissues (Fig. 6). Two tumorous tissue slices per donor and virus strain were infected. By definition, a positive event occurred when one or both slices were positive for viral $\mathrm{N}$ protein expression. A negative event 
was stated, when no viral $\mathrm{N}$ protein was detected in both slices at all. Finally, a proportional calculation between positive and negative events provided the tumor infection rate for each $\mathrm{MeV}$ strain.

As a result, $\mathrm{MeV}-$ Moraten/Edmonston Zagreb and MeV-AIK-C were identified to exhibit the highest tumor infection rate with $75 \%$ positivity of all tested tumor specimens, followed by MeV-Mérieux/Schwarz and MeVL-16 (65\%). Recombinant MeV-GFP particles were found to be less infective on tumorous tissue slices, with tumor infection rates of $45 \%$. Since tumor tissue infections were found to differ in a range of $45-75 \%$, a patient-individualized approach might be of importance for a successful virotherapeutic treatment of primary and secondary tumors of the liver.

\section{Discussion}

Several viruses have a natural potent antitumor activity and it has been shown that we can exploit this natural ability of viruses to directly kill tumor cells alone as well as in combination with other conventional treatment modalities. Therefore, oncolytic viruses are capable of becoming powerful anticancer agents, especially against solid tumors in advanced disease states.

However, it is still a challenging task to maximize oncolytic viral efficacies while ensuring an utmost safety profile of these replication-competent agents. In this context, especially vaccine virus strains outfitted with strong inherent oncolytic properties are of great promise. Measles vaccine viruses $(\mathrm{MeV})$, approved for human use and being applied millions of times with a longstanding excellent safety record, exhibit such a potent natural oncolytic activity together with an ideal safety profile (30).

In the past, a large variety of different $\mathrm{MeV}$ vaccine strains has been generated and is currently in use all over the world. But so far, it is not known whether closely related, but genetically variant $\mathrm{MeV}$ vaccine strains do exhibit significant functional disparities (foremost concerning their oncolytic efficacies as well as safety properties). To address this question, a robust in vitro testing platform is required, which not only comprises immortalized human tumor cells, but also the entirety of non-transformed/non-tumorous human cell types embedded in their natural tissue architectural context. Recently, more and more critical differences between immortalized cell lines, xenograft- and patient-derived specimens have become apparent (31). In addition, cancer is regarded as a complex disease dependent upon the interaction between transformed cells and their surrounding tumor environment, the 'non-cell autonomous constituents' made up of normal parenchymal cells, stromal cells, and immune cells, features that are not represented in the common xenograft approach (32).

These comprehensive requirements are not fulfilled by any in vitro testing which is based only on usage of human tumor cell lines cultivated as monolayers. Thereby, the efficacy of the various oncolytic viruses is assessed only in a non-tissue and non-patient context. As a matter of fact, this current 'monolayer culture standard' is not able to adequately address patient-individual tumor responses and safety features.
Here, we report on the establishment of human precisioncut liver slices (PCLS) as a comprehensive predictive test system for patient-individual tumor response and safety features of oncolytic measles vaccine viruses. Employing primary liver tumor resection specimens, patient-individual tumor and non-tumor characteristics are represented and addressed and the typical patient heterogeneity of clinical study cohorts was reflected also in our in vitro study. Furthermore, due to the generation of multiple, very thin slices from single donor specimens and the availability of slices which had been obtained by successively cutting the same tissue core, we were able to perform serial and repetitional testing on nearly identical material of individual patients (see Figs. 5 and 6: per donor and per virus strain two tumorous tissue slices were infected, respectively). Nevertheless, by definition, usage of such patient-individual clinical grade materials is not compatible with any demands of 'testing object standardization'; evidently, this demand can only be fulfilled when i) immortalized cell lines of clonal origin are used in vitro (7) or ii) when xenograft tumors (usually of clonal origin) are grown in small animal models in vivo (9). In conclusion, the methodology of human precision-cut liver tumor slice testing of oncolytic vectors is able to add novel, patient-individual information on important vector properties thereby enabling a future patient-individual vector preselection for subsequent clinical vector application.

Of note, vitality of resection material is known to differ due to features such as i) pre-treatment with classical chemotherapeutics, biologicals or radiation, ii) central necrosis in large tumors, or iii) prolonged resection surgery procedures. For this reason, we incorporated a receiving quality check for each and every donor sample, so that samples with insufficient viability and consecutively insufficient infectability could be excluded as early as possible from further processing. When complex biological phenomena are to be investigated (i.e. here: target cell infection by oncolytic viruses and subsequent viral gene expression and replication), functional tissue quality tests are preferred over non-functional tests of morphological (morphological tissue assessments, cell number counting) or biochemical nature [determination of cell proliferation or viability by staining with Ki-67 (33), PCNA (34), MTT or MTS $(18,35)$, and measurements of ATP, CYP, LDH, ALT, AST (35)]. Since only viable cells provide an intact machinery for infection and subsequent expression of viral and marker genes, we and others (18) perform infections of PCLS control slices with highly infective recombinant non-replicative adenoviral vectors. Thereby, all steps of target cell infection by oncolytic $\mathrm{MeV}$ particles are simulated in an easy, inexpensive and fast ( $24 \mathrm{~h}$ only) way. Of course, the setting of our quality check approach depends on the infectivity of both tumorous and non-tumorous PCLS control slices for recombinant non-replicative adenoviral vectors. Lack of CAR (adenovirus receptor) expression is known to be a potential cause of intrinsic resistance of target cells to this type of treatment. Whereas non-transformed human liver tissues are well-known for their accessibility to adenoviral infections and their profound expression of the CAR receptor (36), this is not unrestrictedly true for gastrointestinal malignancies (esophageal, pancreatic, colorectal and liver cancer), in which CAR expression was found to vary substantially (37). As a 
consequence, our adenoviral vector-based approach of tissue slice quality checking can only be valid when adenoviral vector-based expression of the GFP marker protein clearly is positive. Therefore, we have decided to exclude all adenoviral transduced GFP-negative tissue slices for further $\mathrm{MeV}$ transduction processing.

On this basis, PCLS slices provide an excellent possiblity to investigate whether or not $\mathrm{MeV}$ particles are able to preferentially infect and replicate in human tumorous tissue sections (thereby mostly sparing surrounding non-tumorous segments). This is of great importance, since we could show that human hepatocytes are permissive to infections with $\mathrm{MeV}$ in vitro (Fig. 2A, second column), albeit at a much lower rate and with a much less pronounced cytopathic effect than immortalized tumor cells of hepatobiliary and gastrointestinal origin.

Employing mixed, tumorous/non-tumorous liver slices from patients exhibiting colorectal liver metastasis for infection by non-targeted MeV-GFP vectors we found a 'natural' tumor area favouring expression pattern of the $\mathrm{MeV}$ encoded GFP marker gene (Fig. 3). However, when employing $\mathrm{MeV}$-infected slices from patients with hepatocellular carcinomas (HCC) which encompass tumorous or nontumorous (mostly fibrotic/cirrhotic) liver segments in a fully separate way (Fig. 5B), Western blot analysis detected comparable amounts of the $\mathrm{MeV} \mathrm{N}$ marker protein in both tumorous or non-tumorous slices in some cases (Fig. 5B, lower left panels: HCC donor 2). Such a reduced 'natural' tumor area favouring expression pattern of $\mathrm{MeV}$ particles in $\mathrm{HCC}$ patients might be, at least in part, the result of an upregulation of $\mathrm{MeV}$ vaccine virus receptor (CD46) expression in nonmalignant fibrotic and cirrhotic tumor-surrounding areas (38).

Since approximately two-thirds of patients with HCC have a reduced liver function because of liver cirrhosis (39), the tumor tissue preference issue of oncolytic $\mathrm{MeV}$ particles is of special awareness in patients with HCC. Recently, knowledge on this topic has been summarized as follows (12): i) MeVinduced liver toxicity was not observed in permissive animal models; ii) studies of hepatic damage by wild-type measles or even $\mathrm{MeV}$ vaccine viruses are extremely rare; iii) posttransplant measles vaccination of patients who have undergone liver transplantation does not result in significant complications and is considered safe. Taken together, these data support the liver safety of oncolytic $\mathrm{MeV}$ particles. Nevertheless, separate clinical toxicology studies in HCC patients employing oncolytic $\mathrm{MeV}$ particles are recommended to impose a special concern to liver toxicology issues.

PCLS slices also provide an excellent possiblity to investigate spreading and penetration capabilities of $\mathrm{MeV}$ in human tumorous and non-tumorous tissue sections, which is of great interest concerning its oncolytic effectiveness. Compared to monolayer cell cultures, the PCLS system is able to give us additional information on virus spread into deeper layers of human multilayer tissue of primary origin. Using a laser scanning confocal microscope we could analyse $\mathrm{MeV}$ infections in detail. In discrepancy to rat liver tissue slices infected with recombinant non-replicating adenovirus which exhibited an infection pattern restricted to the outer layers of the liver slices only (19), we were able to detect $\mathrm{MeV}$ spread throughout whole tissue slices (Fig. 4B) mainly caused by cellular fusions. Provided accessibility to patient tumor materials, from now on this specific feature of oncolytic vectors can be tested in a patient-individual manner, being of great importance for future clinical virotherapy applications.

Furthermore, the PCLS technology also enables the comparative testing of closely related, but genetically variant $\mathrm{MeV}$ vaccine strains (Fig. 6). Currently, a derivative of only a single $\mathrm{MeV}$ vaccine strain, i.e. the $\mathrm{MeV}$ Edmonston $\mathrm{B}$ vaccine strain (14), is employed in clinical phase I trials at the Mayo Clinic in Rochester, MN, USA (http://clinicaltrials.mayo.edu/). For the first time, our study provides results on important tumor infection parameters of four different $\mathrm{MeV}$ vaccine strains and one recombinant $\mathrm{MeV}$-GFP vector tested on nearly identical patient tumorous/non-tumorous tissue slices. In future studies, not only the functional consequences of each and every $\mathrm{MeV}$ vector modification [e.g., targeting modifications (40)] can be investigated in a patient-individual manner, but also of vectors derived from other oncolytic viruses. Thereby, the gap of knowledge on oncolytic vector properties prior to further clinical studies hopefully can be narrowed in the future.

\section{Acknowledgements}

This work was supported in part by grants from the Ministerium für Ernährung und Ländlichen Raum/ Ministerium für Wissenschaft, Forschung und Kunst BadenWürttemberg (MLR/MWK Kap. 0802/98174), the Bundesministerium für Bildung, Wissenschaft, Forschung und Technologie (BMBF 01GU503) and from the Deutsche Forschungsgemeinschaft SFB 773, project C3).

\section{References}

1. Kelly E and Russell SJ: History of oncolytic viruses: genesis to genetic engineering. Mol Ther 15: 651-659, 2007.

2. Li QX, Liu G and Wong-Staal F: Oncolytic virotherapy as a personalized cancer vaccine. Int J Cancer 123: 493-499, 2008.

3. Liu TC, Galanis E and Kirn D: Clinical trial results with oncolytic virotherapy: a century of promise, a decade of progress. Nat Clin Pract Oncol 4: 101-117, 2007.

4. Fielding AK: Measles as a potential oncolytic virus. Rev Med Virol 15: 135-142, 2005

5. Vaha-Koskela MJ, Heikkila JE and Hinkkanen AE: Oncolytic viruses in cancer therapy. Cancer Lett 254: 178-216, 2007.

6. Grote D, Russell SJ, Cornu TI, Cattaneo R, Vile R, Poland GA and Fielding AK: Live attenuated measles virus induces regression of human lymphoma xenografts in immunodeficient mice. Blood 97: 3746-3754, 2001.

7. Peng KW, Ahmann GJ, Pham L, Greipp PR, Cattaneo R and Russell SJ: Systemic therapy of myeloma xenografts by an attenuated measles virus. Blood 98: 2002-2007, 2001.

8. Peng KW, TenEyck CJ, Galanis E, Kalli KR, Hartmann LC and Russell SJ: Intraperitoneal therapy of ovarian cancer using an engineered measles virus. Cancer Res 62: 4656-4662, 2002.

9. Peng KW, Facteau S, Wegman T, O'Kane D and Russell SJ: Non-invasive in vivo monitoring of trackable viruses expressing soluble marker peptides. Nat Med 8: 527-531, 2002.

10. Phuong LK, Allen C, Peng KW, et al: Use of a vaccine strain of measles virus genetically engineered to produce carcinoembryonic antigen as a novel therapeutic agent against glioblastoma multiforme. Cancer Res 63: 2462-2469, 2003.

11. Heinzerling L, Kunzi V, Oberholzer PA, Kundig T, Naim H and Dummer R: Oncolytic measles virus in cutaneous T-cell lymphomas mounts antitumor immune responses in vivo and targets interferon-resistant tumor cells. Blood 106: 2287-2294, 2005.

12. Blechacz B, Splinter PL, Greiner S, et al: Engineered measles virus as a novel oncolytic viral therapy system for hepatocellular carcinoma. Hepatology 44: 1465-1477, 2006. 
13. Christensen LS, Scholler S, Schierup MH, Vestergaard BF and Mordhorst CH: Sequence analysis of measles virus strains collected during the pre- and early-vaccination era in Denmark reveals a considerable diversity of ancient strains. APMIS 110: 113-122, 2002

14. Duprex WP, McQuaid S, Hangartner L, Billeter MA and Rima BK: Observation of measles virus cell-to-cell spread in astrocytoma cells by using a green fluorescent protein-expressing recombinant virus. J Virol 73: 9568-9575, 1999.

15. Parrish AR, Gandolfi AJ and Brendel K: Precision-cut tissue slices: applications in pharmacology and toxicology. Life Sci 57: 1887-1901, 1995.

16. Olinga P, Meijer DKF, Slooff MJH and Groothuis GMM: Liver slices in in vitro pharmacotoxicology with special reference to the use of human liver tissue. Toxicology In Vitro 12: 77-100, 1998.

17. Graaf IA, Groothuis GM and Olinga P: Precision-cut tissue slices as a tool to predict metabolism of novel drugs. Expert Opin Drug Metab Toxicol 3: 879-898, 2007.

18. Kirby TO, Rivera A, Rein D, et al: A novel ex vivo model system for evaluation of conditionally replicative adenoviruses therapeutic efficacy and toxicity. Clin Cancer Res 10: 8697-8703, 2004.

19. Rots MG, Elferink MG, Gommans WM, et al: An ex vivo human model system to evaluate specificity of replicating and non-replicating gene therapy agents. J Gene Med 8: 35-41, 2006.

20. Stoff-Khalili MA, Rivera AA, Le LP, et al: Employment of liver tissue slice analysis to assay hepatotoxicity linked to replicative and nonreplicative adenoviral agents. Cancer Gene Ther 13: 606-618, 2006.

21. van de Bovenkamp M, Groothuis GM, Meijer DK, Slooff MJ and Olinga P: Human liver slices as an in vitro model to study toxicity-induced hepatic stellate cell activation in a multicellular milieu. Chem Biol Interact 162: 62-69, 2006.

22. Elferink MG, Olinga P, Draaisma AL, et al: LPS-induced downregulation of MRP2 and BSEP in human liver is due to a posttranscriptional process. Am J Physiol Gastrointest Liver Physiol 287: G1008-G1016, 2004.

23. Weiss TS, Pahernik S, Scheruebl I, Jauch KW and Thasler WE: Cellular damage to human hepatocytes through repeated application of 5-aminolevulinic acid. J Hepatol 38: 476-482, 2003.

24. Thasler WE, Weiss TS, Schillhorn K, Stoll PT, Irrgang B and Jauch KW: Charitable state-controlled foundation human tissue and cell research: ethic and legal aspects in the supply of surgically removed human tissue for research in the academic and commercial sector in Germany. Cell Tissue Bank 4: 49-56, 2003.

25. Krumdieck CL, dos Santos JE and Ho KJ: A new instrument for the rapid preparation of tissue slices. Anal Biochem 104: 118-123, 1980 .
26. Olinga $\mathrm{P}$, Groen $\mathrm{K}$, Hof $\mathrm{IH}$, et al: Comparison of five incubation systems for rat liver slices using functional and viability parameters. J Pharmacol Toxicol Methods 38: 59-69, 1997.

27. Knipe DM, Howley PM, Griffin DE, Lamb RA, Martin MA Roizman B and Straus SE (eds). Fields Virology. Vol. 1 and 2, 5th edition, Lippincott-Williams and Wilkins, Philadelphia, 2007.

28. Wybranietz WA, Prinz F, Spiegel M, Schenk A, Bitzer M, Gregor M and Lauer UM: Quantification of VP22-GFP spread by direct fluorescence in 15 commonly used cell lines. J Gene Med 1: 265-274, 1999.

29. Bitzer M, Prinz F, Bauer M, et al: Sendai virus infection induces apoptosis through activation of caspase-8 (FLICE) and caspase-3 (CPP32). J Virol 73: 702-708, 1999.

30. Nakamura T and Russell SJ: Oncolytic measles viruses for cancer therapy. Expert Opin Biol Ther 4: 1685-1692, 2004.

31. Newell P, Villanueva A, Friedman SL, Koike K and Llovet JM: Experimental models of hepatocellular carcinoma. J Hepatol 48: 858-879, 2008.

32. Frese KK and Tuveson DA: Maximizing mouse cancer models Nat Rev Cancer 7: 645-658, 2007.

33. Kern MA, Haugg AM, Eiteneuer E, et al: Ex vivo analysis of antineoplastic agents in precision-cut tissue slices of human origin: effects of cyclooxygenase-2 inhibition in hepatocellular carcinoma. Liver Int 26: 604-612, 2006.

34. Wang Y, Thorne S, Hannock J, et al: A novel assay to assess primary human cancer infectibility by replication-selective oncolytic adenoviruses. Clin Cancer Res 11: 351-360, 2005.

35. Lerche-Langrand $\mathrm{C}$ and Toutain HJ: Precision-cut liver slices: characteristics and use for in vitro pharmaco-toxicology. Toxicology 153: 221-253, 2000.

36. Einfeld DA, Schroeder R, Roelvink PW, Lizonova A, King CR, Kovesdi I and Wickham TJ: Reducing the native tropism of adenovirus vectors requires removal of both CAR and integrin interactions. J Virol 75: 11284-11291, 2001.

37. Korn WM, Macal M, Christian C, et al: Expression of the coxsackievirus- and adenovirus receptor in gastrointestinal cancer correlates with tumor differentiation. Cancer Gene Ther 13: 792-797, 2006

38. Kinugasa N, Higashi T, Nouso K, et al: Expression of membrane cofactor protein (MCP, CD46) in human liver diseases. Br J Cancer 80: 1820-1825, 1999.

39. Llovet JM, Fuster J and Bruix J: The Barcelona approach: diagnosis, staging, and treatment of hepatocellular carcinoma. Liver Transpl 10: S115-S120, 2004.

40. Waehler R, Russell SJ and Curiel DT: Engineering targeted viral vectors for gene therapy. Nat Rev Genet 8: 573-587, 2007. 